\title{
Clinical and Bacteriological Profiles of Blood Culture Positive Sepsis in Newborns
}

\author{
Shrestha $\mathrm{P}^{1}$, Das $\mathrm{BK}^{2}$, Bhatta $\mathrm{NK}^{3}$, Jha $\mathrm{DK}^{4}$, Das $\mathrm{B}^{5}$, Setia $\mathrm{A}^{6}$, Tiwari $\mathrm{A}^{7}$ \\ ${ }^{1}$ Dr. P Shrestha Assistant Professor, ${ }^{2}$ Dr. Brija Kishore Das, ${ }^{3}$ Dr. NK Bhatta. ${ }^{4}$ Dr. DK Jha, ${ }^{5}$ Dr. B Das, ${ }^{6}$ Dr. A Setia, Department \\ of Pediatrics and Adolescent Medicine, BP Koirala Institute of Health Sciences (BPKIHS), Dharan, Nepal. ${ }^{7}$ Dr. A Tiwari, \\ Department of Radiodiagnosis, BPKIHS.
}

Address for Correspondence: Dr. P Shrestha, Email: prmd_shrestha@yahoo.com

\begin{abstract}
Neonatal infections currently cause about 1.6 million deaths annually in developing countries ${ }^{1}$. Sepsis and meningitis is responsible for most of these deaths. This study was undertaken to determine the clinical presentations, bacteriological profiles and antibiotic sensitivity patterns of isolates from blood cultures of neonates admitted in a tertiary care hospital in Eastern Nepal. All blood culture reports (n=103) during January 2006 - February 2007 from newborns admitted in neonatal division at BP Koirala Institute of Health Sciences, Nepal were analyzed and antibiotic sensitivity patterns were studied. The positive blood culture was 20\% (103/513). Most (97.1\%) of the sepsis was caused by single organism, while polymicrobial aetiology was observed in $2.9 \%$ cases. Meningitis was documented in $9(8.7 \%)$ cases. Staphylococcus aureus $(38.8 \%)$ and coagulase negative staphylococcus (CONS) $(21.3 \%)$ are the commonest isolates in blood culture. Among gram-negative organisms, Klebsiella species $(11.6 \%)$ and Enterobacter species $(9.7 \%)$ were the leading cause of neonatal sepsis. Majority of newborns with neonatal sepsis presented with refusal to feeds (42.7\%), fever (41.7\%) and jaundice (41.7\%). Most of the organisms showed sensitivity with amino glycosides (gentamicin and amikacin) and third generation cephalosporins. It is concluded that Staphylococcus aureus, CONS, and Klebsiella species remain the principal organisms causing neonatal sepsis and first line antibiotics like amino glycosides should be first choice of drugs.
\end{abstract}

Key words: Blood culture, neonatal sepsis, antibiotic sensitivity.

\section{Introduction}

Neonatal sepsis is a significant cause of neonatal morbidity and mortality in the newborn, particularly in preterm, low birth weight infants ${ }^{2,3}$. According to World Health Organization (WHO) estimates, neonatal sepsis remains the major cause out of five million neonatal deaths per year ${ }^{4}$. The spectrum of organisms that causes neonatal sepsis changes over times and varies from region to region. This is due to the changing pattern of antibiotic use and changes in life style.

Reports of the epidemiology of neonatal sepsis from Nepal are few. The epidemiological data from other developing countries, however, shows important differences in the incidence, risk factors, pattern and antimicrobial sensitivities of pathogens and mortality from that of developed countries ${ }^{5,6,7}$. Group B streptococcal disease is the most important cause of neonatal sepsis in Europe and North America $^{8}$ but there is a preponderance of gram-negative organisms in tropical and developing countries ${ }^{9}$. This study was conducted to determine the clinical presentations, bacteriological profiles and antibiotic sensitivity patterns of isolates from blood cultures of neonates admitted in a tertiary care hospital in eastern region of Nepal.

\section{Materials and Methods}

This retrospective study includes 513 cases of clinically suspected neonatal sepsis admitted in Neonatal Division, during the period of January 2006 to February 2007 at BP Koirala Institute of Health Sciences, Dharan, Nepal. This is the largest tertiary care pediatric hospital in Eastern region of Nepal. Diagnosis of neonatal sepsis was based upon the antenatal high risk factors and signs and symptoms of sepsis. Neonatal sepsis was suspected in the following conditions:

At birth: All newborns (i) born to mothers with maternal fever, or prolonged rupture of membrane ( $>18$ hours), or foul smelling or meconium stained liquor, or frequent unclean vaginal examination ( $>3$ ), and/or (ii) having severe prematurity or birth asphyxia necessitating active resuscitation.

After birth: All neonates with lethargy, refusal to feeds, abdominal distention, respiratory distress, temperature instability (hypothermia/fever), pathological jaundice, seizures, vomiting, and autonomic dysfunction.

Following procedures: All newborns undergone exchange transfusion.

Blood samples were collected in all cases for culture and sensitivity studies. Lumbar puncture for CSF analysis was done in suspected cases of meningitis. All blood culture reports were analyzed and those neonates with positive culture reports were evaluated. All culture positive cases were further divided into early onset sepsis (EOS) and late onset sepsis (LOS), on the basis of time of clinical presentation. Those presented within first 72 hours of life were diagnosed as EOS and after 72 hours of life as LOS.

Statistical analysis was done to evaluate the significant differences between LOS and EOS by using SPSS 11 version. 
Chi-square test and Fisher's Exact test were used to determine the significant difference.

\section{Results}

Total of 513 newborns with clinical sepsis were admitted between the period of January 2006 and February 2007. Blood culture reports were positive in 103 cases (20\%). Out of 103 cases, 66.9\% $(n=69)$ had LOS and 33.1\% $(n=34)$ had EOS. Majority (68.9\%) of newborns were delivered at home $(n=71)$. Male to female ratio was $3.1: 1$. The patients' characteristic is depicted in Table 1.

Table 1: Patients Characteristic with Neonatal Sepsis

\begin{tabular}{|c|c|c|c|}
\hline & Total(n=103) & $\begin{array}{c}\text { EOS } \\
(\mathbf{n = 3 4 )}\end{array}$ & $\begin{array}{c}\text { LOS9 } \\
(\mathbf{n}=\mathbf{6 9})\end{array}$ \\
\hline Gender & & & \\
\hline Male & 78 & 27 & 51 \\
\hline Female & 25 & 7 & 18 \\
\hline Male: Female & $3.1: 1$ & $3.8: 1$ & $2.8: 1$ \\
\hline Gestation & & & \\
\hline Preterm & 25 & 6 & 19 \\
\hline Term & 75 & 26 & 49 \\
\hline Post-term & 3 & 2 & 1 \\
\hline Birth weight (g) & & & \\
\hline$<1500$ & 11 & 27 & 65 \\
\hline $1500-2500$ & 3 & 8 & 23 \\
\hline$>2500$ & 8 & 19 & 42 \\
\hline Mode of delivery & & & \\
\hline SVD & 76 & 27 & 25 \\
\hline LSCS & 9 & 51 & 18 \\
\hline
\end{tabular}

Majority of newborns with neonatal sepsis presented with refusal to feeds $(42.7 \%)$, fever $(41.7 \%)$, and jaundice $(41.7 \%)$. Though statistically not significant, respiratory distress was more pronounced in EOS than in LOS $(35.3 \%$ Vs $26.1 \%$ ), whereas seizures were more common in LOS (23.2\%). Two newborns with seizures in EOS group had biochemical evidence of meningitis with CSF culture sterile in both these babies. In LOS group, 7 newborns had meningitis with 4 CSF culture positive (Klebsiella species in 2 cases, and 1 each Enterobacter species and Acinetabacter). Table 2 shows various clinical presentations of neonatal sepsis. There were no significant differences in clinical presentations between LOS and EOS.

Staphylococcus aureus (S aureus) and coagulase negative staphylococcus (CONS) are the commonest pathogens causing both EOS and LOS. Among gram-negative organisms, Klebsiella species (11.6\%) and Enterobacter species (9.7\%) are the leading cause of neonatal sepsis. Association with 2 organisms was seen in 3 cases $(2.9 \%)$. E coli and Klebsiella were observed in two cases and $\mathrm{S}$ aureus and citrobacter were seen in one case. Isolates grown in blood culture is depicted in Table 3. Table 4 shows the antibiotic sensitivity of the isolates (in percentage) to the commonly used antibiotics.

Table 2: Clinical Presentations of Neonatal Sepsis

\begin{tabular}{|c|c|c|c|c|c|c|}
\hline \multirow{2}{*}{ Clinical features } & \multicolumn{2}{|c|}{ Total $(n=103)$} & \multicolumn{2}{|c|}{$\operatorname{EOS}(n=34)$} & \multicolumn{2}{|c|}{$\operatorname{LOS}(n=69)$} \\
\hline & $\mathbf{n}$ & $\%$ & $\mathbf{n}$ & $\%$ & $\mathbf{n}$ & $\%$ \\
\hline Refusal to feed & 44 & 42.7 & 18 & 52.9 & 26 & 37.7 \\
\hline Fever & 43 & 41.7 & 11 & 32.3 & 32 & 46.4 \\
\hline Jaundice & 43 & 41.7 & 13 & 38.2 & 30 & 43.4 \\
\hline Respiratory distress & 30 & 29.2 & 12 & 35.3 & 18 & 26.1 \\
\hline Seizures & 22 & 21.4 & 6 & 17.6 & 16 & 23.2 \\
\hline Lethargy & 15 & 14.6 & 5 & 14.7 & 10 & 14.5 \\
\hline Hypothermia & 7 & 6.8 & 4 & 11.7 & 3 & 4.4 \\
\hline Vomiting & 7 & 6.8 & 2 & 5.8 & 5 & 7.3 \\
\hline Abdominal distension & 5 & 4.8 & 0 & 0 & 5 & 7.3 \\
\hline
\end{tabular}


Table 3: Organisms Isolated From Blood Culture

\begin{tabular}{|l|c|c|c|c|c|c|}
\hline \multirow{2}{*}{ Organisms } & \multicolumn{2}{|c|}{$\begin{array}{c}\text { Total } \\
(\mathbf{n = 1 0 3})\end{array}$} & \multicolumn{2}{c|}{$\begin{array}{c}\text { EOS } \\
(\mathbf{n}=\mathbf{3 4})\end{array}$} & \multicolumn{2}{c|}{$\begin{array}{c}\text { LOS } \\
(\mathbf{n = 6 9 )}\end{array}$} \\
\cline { 2 - 7 } & $\mathbf{n}$ & $\mathbf{\%}$ & $\mathbf{n}$ & $\mathbf{\%}$ & $\mathbf{n}$ & $\%$ \\
\hline S. aureus & 40 & 38.8 & 12 & 35.3 & 28 & 40.5 \\
\hline CONS & 22 & 21.3 & 7 & 20.6 & 15 & 21.7 \\
\hline $\begin{array}{l}\text { Klebsiella } \\
\text { species }\end{array}$ & 12 & 11.6 & 4 & 11.7 & 8 & 11.4 \\
\hline $\begin{array}{l}\text { Enterobacter } \\
\text { species }\end{array}$ & 10 & 9.7 & 3 & 8.8 & 7 & 10.2 \\
\hline E. coli & 7 & 6.7 & 3 & 8.8 & 4 & 5.8 \\
\hline Mixed & 3 & 2.9 & 1 & 2.9 & 2 & 2.9 \\
\hline Acinetabacter & 2 & 1.9 & 2 & 5.8 & 0 & 0 \\
\hline Enterococcus & 2 & 1.9 & 0 & 0 & 2 & 2.9 \\
\hline $\begin{array}{l}\text { Streptococcus } \\
\text { species }\end{array}$ & 2 & 1.9 & 0 & 0 & 2 & 2.9 \\
\hline Others & 3 & 2.9 & 2 & 5.8 & 1 & 1.4 \\
\hline
\end{tabular}

Table 4: Antibiotics Sensitivity Patterns in Common Isolates

\begin{tabular}{|l|c|c|c|c|c|c|}
\hline \multirow{2}{*}{ Organisms } & \multicolumn{2}{|c|}{$\begin{array}{c}\text { Total } \\
(\mathbf{n = 1 0 3})\end{array}$} & \multicolumn{2}{c|}{$\begin{array}{c}\text { EOS } \\
(\mathbf{n = 3 4 )}\end{array}$} & \multicolumn{2}{c|}{$\begin{array}{c}\text { OOS } \\
(\mathbf{n = 6 9 )}\end{array}$} \\
\cline { 2 - 7 } & $\mathbf{n}$ & $\mathbf{\%}$ & $\mathbf{n}$ & $\%$ & $\mathbf{n}$ & $\%$ \\
\hline S. aureus & 40 & 38.8 & 12 & 35.3 & 28 & 40.5 \\
\hline CONS & 22 & 21.3 & 7 & 20.6 & 15 & 21.7 \\
\hline $\begin{array}{l}\text { Klebsiella } \\
\text { species }\end{array}$ & 12 & 11.6 & 4 & 11.7 & 8 & 11.4 \\
\hline $\begin{array}{l}\text { Enterobacter } \\
\text { species }\end{array}$ & 10 & 9.7 & 3 & 8.8 & 7 & 10.2 \\
\hline E. coli & 7 & 6.7 & 3 & 8.8 & 4 & 5.8 \\
\hline Mixed & 3 & 2.9 & 1 & 2.9 & 2 & 2.9 \\
\hline Acinetabacter & 2 & 1.9 & 2 & 5.8 & 0 & 0 \\
\hline Enterococcus & 2 & 1.9 & 0 & 0 & 2 & 2.9 \\
\hline $\begin{array}{l}\text { Streptococcus } \\
\text { species }\end{array}$ & 2 & 1.9 & 0 & 0 & 2 & 2.9 \\
\hline Others & 3 & 2.9 & 2 & 5.8 & 1 & 1.4 \\
\hline
\end{tabular}

\section{Discussion}

For the effective management of neonatal sepsis, knowledge about bacteriological profiles and antibiotic sensitivity patterns play a vital role. In our study, we found that LOS was more common than EOS, in contrast to other reports where EOS was more common ${ }^{10,11}$. However our finding is consistent with the study where LOS was more common than $\mathrm{EOS}^{12}$. This discrepancy could be due to the fact that mortality in early-onset cases is relatively high ${ }^{13}$. Males have been reported to be 2 to 5 times more than females to develop neonatal sepsis ${ }^{13}$. The male to female ratio of $3.1: 1$ in our study is in agreement with the above study, may be due to a gender bias in presentation to hospital for care.

In this study, blood culture positivity rate is $20 \%$, whereas in $80 \%$ cases there was no growth. This finding is comparable with other reports ${ }^{14,15}$. A low blood culture isolation rate could be due to administration of antibiotic before blood collection from the primary centers or the possibility of infection with anaerobes. A negative blood culture does not exclude sepsis and about $26 \%$ of all neonatal sepsis could be due to anaerobes ${ }^{16}$. In this study the predominant isolates was $\mathrm{S}$ aureus which is in agreement with other reports ${ }^{17,18}$.In this study, Klebsiella species (11.6\%) and enterobacter species $(9.7 \%)$ are the leading cause of neonatal sepsis among gram negative organisms. The report of the National NeonatalPerinatal database showed Klebsiella as the predominant $(29 \%)$ pathogen ${ }^{19}$. The clinical significance of relatively low virulence isolates, such as CONS and Enterococcus is difficult to ascertain. These organisms can cause true bacteremia or their isolation may represent simple contamination. It would be unfair to ignore such isolates as contaminants.

Most of the organisms are sensitive to aminoglycosides (Amikacin and gentamicin) and third generation cephalosporins. In general, the sensitivity of the gramnegative isolates to gentamicin supports continued use of this agent in the initial, empiric treatment of neonatal sepsis in our hospital, and also supports WHO recommendations that management of young infants up to age 2 months include parenteral use of benzyl penicillin or ampicillin plus an aminoglycoside such as gentamicin ${ }^{20}$.

\section{Conclusion}

Thus, it is concluded that $\mathrm{S}$ aureus, CONS, and gram negative organisms (Klebsiella, Enterobacter and E. coli) are the leading cause of neonatal sepsis in Nepal and most of them are sensitive to aminoglycosides and third generation cephalosporins.

\section{References}

1. Vergnano S, Sharland M, Kazembe P, Mwansambo C, Heath PT. Neonatal Sepsis: An International; Perspective. Arch Dis Child Fetal Neonatal. 2005; 90: F220-24

2. Stoll BJ, Hansen N. Infections In VLBW Infants: Studies From The NICHD Neonatal Research Network. Semin Perinatol. 2003; 27: 293-301

3. Stoll BJ, Hansen NI, Adams-Chapman I et al. Neurodevelopmental And Growth Impairment Among Extremely Low-Birth-Weight Infants With Neonatal Infections. JAMA. 2004; 292: 2357-65

4. WHO. Perinatal Mortality. Report No: WHO/FRH/ MSM/967. Geneva: WHO, 1996

5. Darmstadt GL, Black RE, Santosham M. Research Priorities And Postpartum-Care Strategies For The Prevention And Treatment Of Neonatal Infection In Less Developed Countries. Pediatr Infect Dis J 2000; 19: 739-50. 
6. Polin RA, St. Geme JW III. Neonatal Sepsis. Adv Paediatr Infect Dis 1992; 7: 25-61.

7. WHO. Young Infants Study Group. Bacterial Etiology Of Serious Infections In Young Infants In Developing Countries: Results Of A Multicenter Study. Pediatr Infect Dis J 1999; 18: S17-22.

8. Fisher G, Horton RE, Edelman R. Summary Of The Neonatal Institute Of Health Workshop On Group B Streptococcal Infections. J Infect Disease 1983; 148: 163-6.

9. Sharma PP, Halder D, Dutta A. Bacteriological Profile Of Neonatal Septicemia. Indian Pediatr 1987; 11: 1010-7.

10. Fisher G, Horton RE, Edelman R. Summary Of The Neonatal Institute Of Health Workshop On Group B Streptococcal Infections. J Infect Disease 1983; 148: 163-6

11. Glandstone IM, Ehrenkranz RA, Edberg SC, Baltimore RS. A Ten Year Review Of Neonatal Sepsis And Comparison With The Previous Fifty Year Experience. Pediatr Infect Dis J 1990; 9: 819-25.

12. Nawshad Uddin Ahmed A.S.M., Azad Chowdhury M.A.K., Hoque M, Darmstadt GL. Clinical And Bacteriological Profile Of Neonatal Septicemia In A Tertiary Level Pediatric Hospital In Bangladesh. Indian Pediatr 2002; 39:1034-9

13. Bhutta ZA, Naqvi SH, Muzzaffar T, Farooqui J. Neonatal Sepsis In Pakistan: Presentation And Pathogen. Acta Pediatr Scand 1991; 80: 596-601.
14. Mathur $M$, Shah $H$, Dixit $K$, Khambaskone $S$, Chakrapani A, Irani S. Bacteriological Profile Of Neonatal Septicemia Cases (For The Year 1990-91). J Post grad Med 1994; 40(1): 18-20

15. Mondal GP, Raghvan M, Bhat BV. Neonatal Septicemia Among Inborn And Outborn Babies In A Referral Hospital. Indian J Pediatr 1991; 58: 529-33

16. Chow AW, Leake RD, Yamanchi T. The Significance Of Anaerobes In Neonatal Bacteremia. Analysis Of 23 Cases And Review Of Literature. Pediatrics 1974; 54; 736-45

17. Karthikeyan G, Premkumar K. Neonatal Sepsis: Staphylococcus Aureus As The Predominant Pathogen. Indian J Pediatr 2001; 68: 715-7

18. Thomas M, Padmini B, Srimathi G, Sundararajan V, Rajni BA. Microbial Profile Of Neonatal Infection In Coimbatore. Indian J Pediatr 1999; 66: 11-4

19. Vital Statistics: Neonatal morbidity and mortality; report of the National neonatal-Perinatal database. Indian Pediatr 1997; 34: 1039-42

20. World Health Organization. Acute respiratory infections in children: case management in hospitals in developing countries. Geneva: WHO, 1990; WHO/ARI/90.5. 